\title{
Design and Development of the Engine Unit for a Twin-Rotor Unmanned Aerial Vehicle
}

\author{
G. Avanzini, S. D’Angelo, G. de Matteis
}

Advanced computer-aided technologies played a crucial role in the design of an unconventional Uninhabited Aerial Vehicle (UAV), developed at the Turin Technical University and the University of Rome "La Sapienza". The engine unit of the vehicle is made of a complex system of three two stroke piston engines coupled with two counter-rotating three-bladed rotors, controlled by rotary PWM servos. The focus of the present paper lies on the enabling technologies exploited in the framework of activities aimed at designing a suitable and reliable engine system, capable of performing the complex tasks required for operating the proposed rotorcraft. The synergic use of advanced computational tools for estimating the aerodynamic performance of the vehicle, solid modeling for mechanical components design, and rapid prototyping techniques for control system logic synthesis and implementation will be presented.

Keywords: uninhabited aerial vehicle, computational fluid dynamics, solid modeling, rapid prototyping.

\section{Introduction}

The last decade saw ever growing interest in the academic world in the study of autonomous flight. Most of the academic projects share a common approach, where an existing model aircraft is equipped with sensors and on-board computers, in order to achieve the prescribed level of autonomous management of the mission profile. As for aircraft with vertical take-off and landing (VTOL) capabilities, model helicopters such as the Yamaha R-max and R-50 were employed by the University of California at Berkeley [1], Carnegie Mellon University [2] and the Georgia Institute of Technology [3].

A more ambitious project has been in progress in the past 5 years at the Department of Aerospace Engineering of the Polytechnic of Turin (DAE) and the Department of Mechanics and Aeronautics of the University of Rome "La Sapienza" (DMA), where the vehicle itself is a completely new machine, characterized by an original configuration, and all the activities related to the design, development, ground and flight testing of the vehicle are performed in an academic framework. The program received funding from the Italian National Plan for Research in Antarctica (PNRA) and the Italian Ministry for Education, Scientific Research and Innovation (MIUR), and it was entirely carried on in academic structures, with limited support from small and medium-size enterprises, for the realization of those mechanical, electrical and electronic components that could not be built in the workshops of the involved universities.

The shrouded fan uninhabited aerial vehicle (UAV), shown in Fig. 1, is made of a toroidal fuselage that envelopes two counter-rotating, three bladed rotors, driven by three two-stroke piston engines, derived from a model used for para-gliders. A brief description of the vehicle is reported in the next paragraph, while its dimensions and expected performance are reported in Tab. 1. More details on the vehicle itself, on the status of the project and related activities can be found in [4] and [5]. Next, the advanced tools employed for the design, development and testing of the engine unit will be presented. The three sections will deal with: (i) aerodynamic modeling of the shrouded fan configuration, using advanced CFD software;

(ii) solid modeling of the mechanical components, using CAD software;

(iii) rapid prototyping techniques for the development of the control system.

As for the last point, details will be given on the control system for engine rpm control currently being tested on the vehicle prototype. A section of conclusions ends the paper.

\section{2 "Ro.W.En.A." (ROtorcraft With ENhanced Autonomy)}

As shown in Fig. 1, the vehicle is characterized by an axial-symmetric configuration, which allows for rotations around the symmetry axis in any flight condition. Moreover, the fuse-

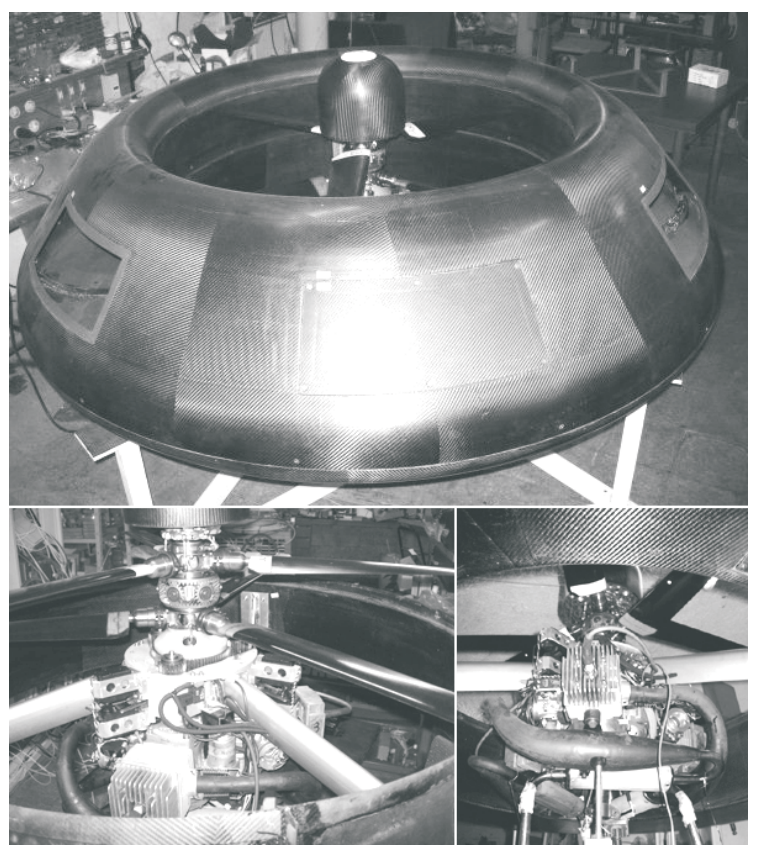

Fig. 1: Prototype of the UAV 
Table 1: UAV characteristics and expected performance

\begin{tabular}{|l|l|}
\hline External diameter: & $1.9 \mathrm{~m}$ \\
\hline Rotor diameter: & $1.1 \mathrm{~m}$ \\
\hline Hub diameter: & $0.25 \mathrm{~m}$ \\
\hline Max. power at $11000 \mathrm{rpm}:$ & $42 \mathrm{hp}$ \\
\hline Rotor angular velocity: & $3000 \mathrm{rpm}$ \\
\hline Maximum TO weight: & $800 \mathrm{~N}$ \\
\hline Payload: & $100 \mathrm{~N}$ \\
\hline Maximum speed: & $30 \mathrm{~ms}^{-1}$ \\
\hline Endurance: & $1.5 \mathrm{~h}$ \\
\hline Service ceiling: & $2000 \mathrm{~m}$ \\
\hline
\end{tabular}

lage sizably improves rotor performance in hovering and at low-speed [6], while protecting them in the case of low-speed impacts against obstacles, thus increasing the safety level for nearby personnel during ground operations with respect to a more conventional rotary-wing configuration with unprotected rotors. These features will provide the vehicle with unique maneuvering capabilities in a confined space and sensor aiming during forward flight. The drawbacks of such a configuration are (i) a relevant penalty in terms of performance in forward flight, due to the pitch-down attitude necessary to maintain a trim condition at high speed, with an increase of frontal area and, thus, of the total drag, and (ii) the inherent instability of the unaugmented aircraft dynamics, due to the absence of any stabilizing surface, as discussed in detail in [6] and [7].

There are some other vehicles, such as the iSTAR MAV [8], that exploit the benefits of the ducted fan. Nonetheless the iSTAR MAV is significantly different, inasmuch as it uses a ducted, constant pitch propeller for thrust generation, thrust modulation being achieved by engine rpm control, and control moments being generated by vanes in the propeller wake. Conversion to an airplane-like attitude is necessary for high-speed flight and the (usually small) payload is placed in the central hub. The only vehicle of comparable size with a configuration similar to that of "Ro.W.En.A" is the Sikorsky Cypher [9], but many distinctive features make the rotorcraft presented in this paper a completely original machine. The Cypher is devoted primarily to military uses and, in this sense, it was designed as an expendable, single engine vehicle. By contrast, the UAV currently under development at DAE and DMA is aimed at civil and/or scientific applications, where the issue of safety for both payload and surrounding personnel is a major concern. In this framework, the design of a small-size, multi-engine machine, capable of performing one-engine-out emergency landings is far from being a trivial task. Moreover, a vehicle developed industrially can rely upon the most advanced technologies, such as the Advancing Blade Concept (ABC), for solving the problem of the retreating blade stall at high speed, and elastomeric joints for blade pitch control, technologies that are ruled out in a research program developed under the tight budget constraints typical of an academic framework.

As for the mechanical assembly, the engine unit is probably the most complex, wholly original component of the vehicle. This unit is made of three two-stroke, air cooled piston engines, coupled with two counter-rotating, three-bladed rotors with composite, rigid blades. The engines are mounted on a titanium mount, and lie downstream of the rotor, in order to maximize the cooling effect of the wake, thus avoiding the complexity and weight of a liquid cooling system. Each engine is connected by its own drive train to the main rotor gear. Inside the pinion, a system of clutch and free wheels will isolate the failed engine, as soon as it does not deliver torque to the main gear, in order to avoid the possibility that the failure of one of the engines stops the other two. The maximum power delivered by two engines should be sufficient for safely landing the rotorcraft, as demonstrated by the power requirement estimate of [6]. The main gear drives the lower rotor, while the upper rotor is driven by the reverse gear. With this configuration, no rotating shaft is present and this, in turn, results in a reduced vibration level, a feature that was experimentally verified during the ground testing of the rotors described in [4], up to the nominal rotor speed of 3,000 rpm.

The control of thrust and moments generated by the shrouded rotors is achieved by means of two independent, helicopter-like swashplates, the position of which is determined by six couples of rotary digital PWM servos. The carbon fiber blades are mounted on steel pivots, which must be capable of standing both a very high static load due to the centrifugal force acting on the blades and the high frequency dynamic loads due to blade pitch cyclic variation. The high frequency load also challenges the electrical servos. In this respect, digitally controlled PWM servos can stand such a load only if used in a coupled configuration. If on the one hand, this choice causes a considerable reduction of the overall system reliability, on the other it allowed us to go on with the research program without the development of an ad-hoc micro-actuator, an activity that, although representing on its own an interesting field for the application of advanced design technologies, was considered not strategic at the current status of the project, where the in-flight demonstration of the vehicle concept is considered the main objective.

In this respect, the development of a vehicle suitable for civil applications, accompanied by the requirement for the capability of performing an emergency landing in case of engine failure, was already a very ambitious scope. The multi-engine configuration represents a sizeable penalty in terms of power-to-weight ratio and overall system complexity, from both the mechanical and avionic system point of view. Also, engine start-up and rotor speed control become more complicated, because it is necessary to properly coordinate the control action on three engine throttles, while keeping the power delivered and the fuel consumption of the three engines close one to the others. The mechanical complexity of the engine mount, drive trains and blade pitch control system is accompanied by the uncertainty relative to the vehicle dynamics, the configuration of which is considerably different from that of any other conventional rotorcraft. Unfortunately, Sikorsky delivered to the public literature only qualitative data on the Cypher, and a detailed study, presented in [6], was necessary for determining a reasonable, although uncertain, vehicle model and for studying its dynamic behavior.

It was only thanks to the modern advanced design and computational tools described in the sequel that the enabling 
technologies required for developing such a complex vehicle were made available to Faculty staff, technicians and students, allowing for surprising advances in the project, in spite of very limited human and economic resources.

\section{Aerodynamic design with advanced CFD tools}

Budget constraints ruled out from the beginning of the research program the possibility of wind-tunnel tests on a scale model of the shrouded-fan UAV, the cost of development of an instrumented model being close to that of the full size prototype. Computational fluid dynamics software was regarded as a viable solution for obtaining a reasonable estimate of the aerodynamic behavior of the vehicle in a short time, with the possibility of "testing" several rotor-fuselage configurations, in the course of a few hours.

During the first phase of the program, i.e., vehicle preliminary sizing and configuration design, the main problems were the estimate of rotor performance and power demand in every flight condition, and the determination of the aerodynamic model of the vehicle to be used for flight simulation of the UAV and for synthesizing suitable control laws.

In this phase the numerical code VSAERO, by Analytical Methods, Inc., was used [10]. This software uses a boundary element integral method for potential flow solution, with boundary layer correction. An iterative wake relaxation

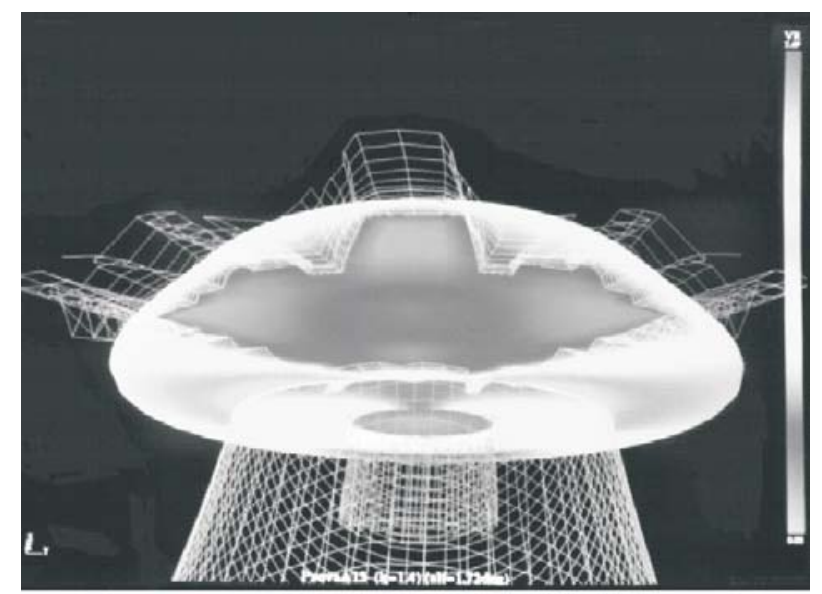

Fig. 2: Graphical output of CFD code VSAERO ${ }^{\text {TM }}$ scheme is used for determining flowfields about lifting and blunt bodies. Overall aerodynamic force and moment coefficients can be easily determined at the end of the computation, from the pressure distribution over the body. An example of the results obtained from the graphical output of VSAERO is reported in Fig. 2.

The numerical code is extremely efficient, from the computational point of view, being able to provide a solution on a common desktop computer in just a few minutes. Nonetheless, the application was not straightforward for the present configuration, inasmuch as (i) the rotor-fuselage interaction is extremely complex and (ii) the wake separation can be automatically detected only in two dimensional flows, or when sharp edges clearly indicate a reasonable separation point, a feature not present in a doughnut-shaped fuselage.

As for the first issue, a strong simplifying assumption was employed, such that the rotors were modeled as an actuator disk with assigned pressure increment. In this way, the simulated flow-field took into account the presence of the rotors, when determining the pressure distribution over the fuselage. By contrast, the aerodynamic model of the rotors, based on a simple strip-theory, did not take into account the influence of the fuselage on the rotor inflow. The inflow is approximated by a triangular velocity distribution determined iteratively on the basis of a momentum conservation argument.

Wake separation points are relatively easy to detect when axial flow is considered, but the problem is much more complicated when forward flight is dealt with. A reasonable estimate of wake separation points was made on the basis of a pressure gradient evaluation for the potential solution and data obtained from published experiments with flow visualizations of separation from doughnut-shaped bodies. In this sense some recent studies made at the Georgia Institute of Technology were of great help in understanding the nature of the closed wake downstream the fuselage.

An aerodynamic database was obtained with VSAERO code, where lift, drag and pitch moment coefficients are tabulated as a function of fuselage angle of attack, for different values of the inflow parameter

$$
k=\frac{V}{\left(v_{i}-w\right)},
$$

where $\boldsymbol{V}=(u, v, w)^{\mathrm{T}}$ is the velocity vector expressed in body axis components, $V=\|\boldsymbol{V}\|$ and $v_{i}$ is the velocity increment induced by the rotor. Details on the aerodynamic model de-
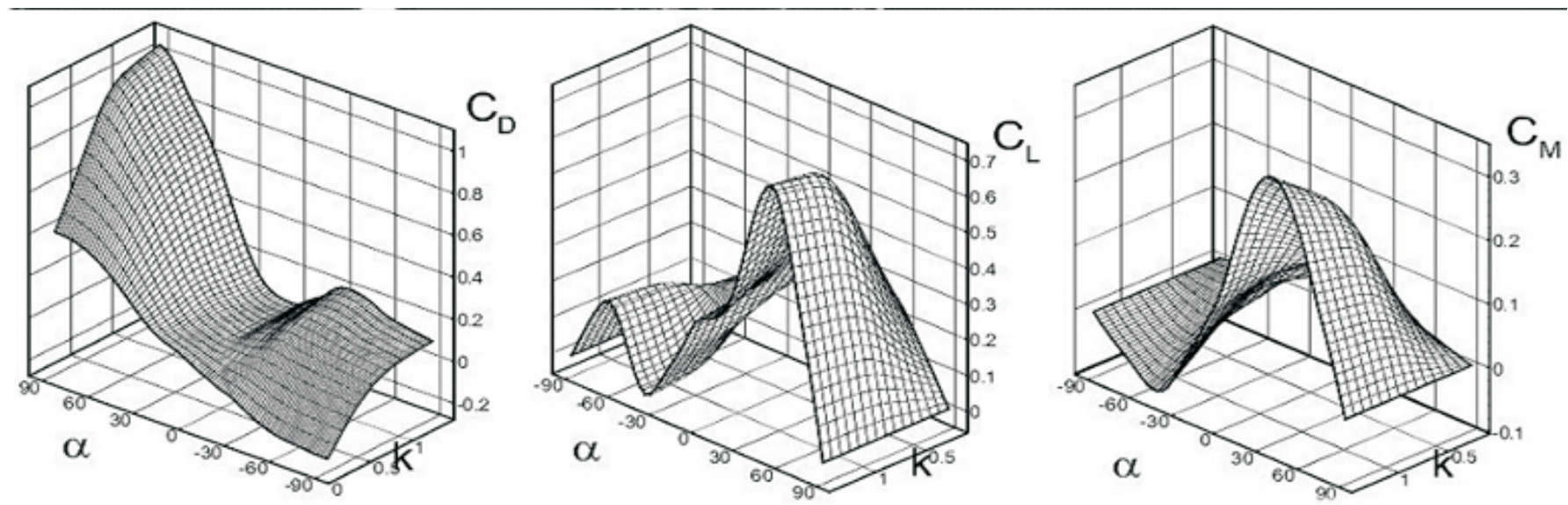

Fig. 3: Plot of aerodynamic force and moment coefficient acting on the UAV fuselage 

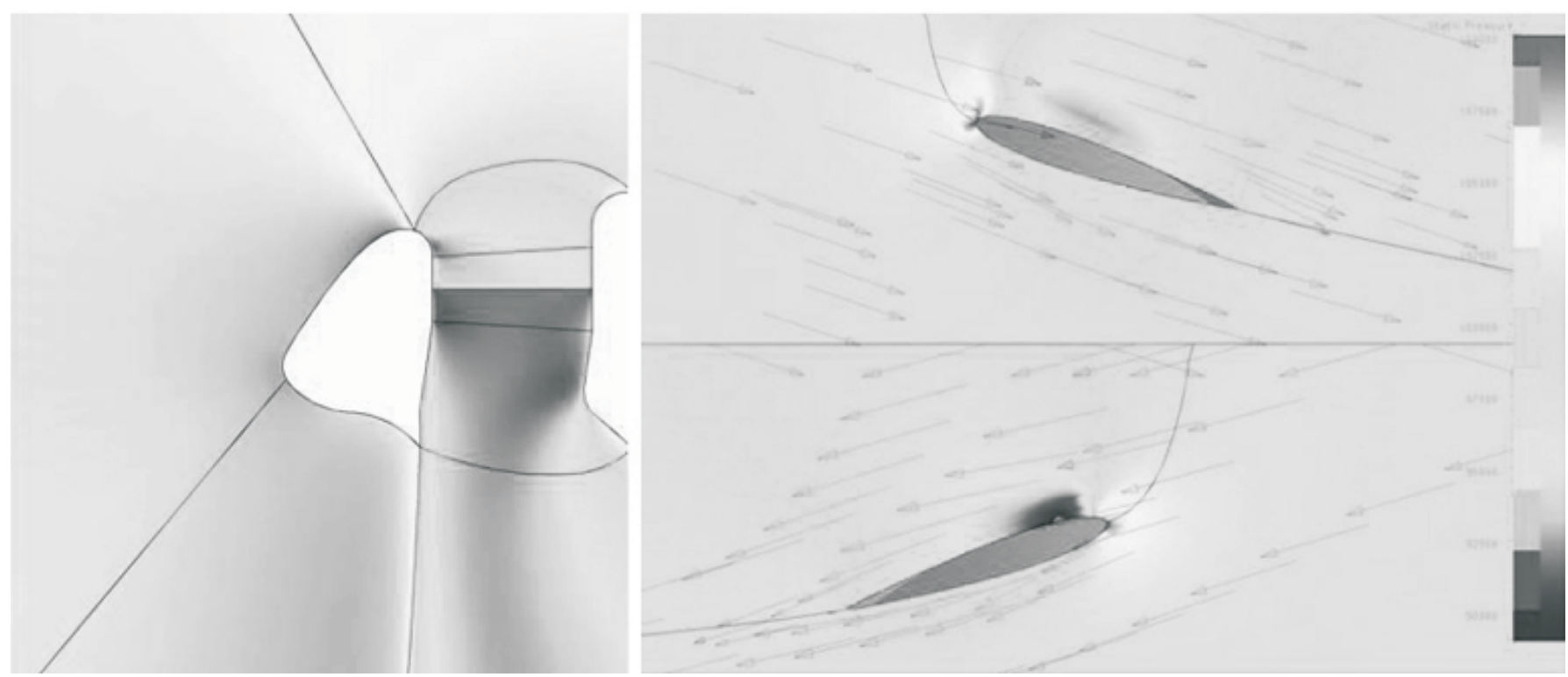

Fig. 4: Examples of Fine/TURBO graphical output

veloped in this phase (and still used in the simulation model of the UAV) can be found in [11]. Plots of the aerodynamic coefficients are reported in Fig. 3.

A second set of CFD simulations was performed using one of the most advanced, sophisticated, and complex flow solvers available on the market, the full Navier-Stokes code FINE/Turbo, developed by NUMECA International s.a. This package provides the user with the possibility of solving very complex flowfields, with reliable automatic mesh generation [12]. Of course, the code is considerably more computationally demanding and, for this reason, its use for realizing a new database was not considered, inasmuch as several machine-months would have been necessary for completing such a job.

This code was used in order to define the final configuration of the fuselage, in terms of section shape, and to investigate in greater detail phenomena not modeled by the Euler solver of VSAERO, such as the flowfield between the rotors, rotor-fuselage interaction, and wake separation from the fuselage in hovering, axial translation and forward flight. In the latter case, it was necessary to model the rotors like an actuator disk, inasmuch as the solver can deal with unsteady periodic solutions only in axial flow. In this way it was also possible to make a comparison with the results obtained from the potential code in a given set of working points. Although this comparison cannot be considered a true validation of the aerodynamic database determined by VSAERO, the combined use of a simple and efficient potential code with a more sophisticated Navier-Stokes solver significantly improved the confidence in the obtained aerodynamic model of the vehicle. Two of the most important results obtained from the analysis performed with the FINE/Turbo software are the determination of the power required by the rotor system and rotor efficiency in hovering, two figures that play a crucial role in engine sizing and vehicle performance estimation. Required power of almost $21 \mathrm{~kW}$ (28 hp) was evaluated, and the beneficial effect of the toroidal fuselage at low speed received sound confirmation, when fuselage lift at hovering (due to the suc- tion effect at the shroud inlet) was determined to be as high as $1 / 3$ of the total rotor thrust.

\section{CAD and solid modeling}

The design of the mechanical components also relied heavily on the most advanced tools for Computer Aided Design and solid modeling. In the very first phase of the project, Autocad, by Autodesk Inc., demonstrated serious limitations when dealing with the design of such a complex machine. As an example, it was not possible to check for interferences in the mechanism for controlling blade pitch by rotor swashplates. Solid modeling became necessary also for automatically generating the surfaces of those components that were to be realized by numerically controlled milling machines. For this reason, licenses for the use of SolidWorks, by Solid Works Corp., were acquired and, later, for Unigraphics, by UGS PLM International.

Together with the aforementioned capabilities, parametric modeling of mechanical components allows for a trial-and-error approach, which would otherwise be extremely time consuming, where several mechanism configurations and dimensions of different components can be varied arbitrarily, until an optimal configuration is found that satisfies all the requirements. Mechanical components interference is ruled out at the design stage, by virtually moving all the components in the assembled mechanism. Finally, the interface with the most widely used finite element codes, such as Nastran, by MSC Software Corp., allows for a user-friendly environment, where also structural analysis is carried out efficiently. Instead of going into greater detail with the presentation of the functionalities of solid modeling software, which can be found in any manual of the aforementioned software, their capabilities on the present project are demonstrated by Fig. 5, which represents the complex linkages that drive the rotor swashplates in order to control blade pitch according to the PWM servo commands.

Every part of the vehicle and every piece of equipment developed to ground test the machine was designed, modeled and realized on the basis of a detailed study carried out 


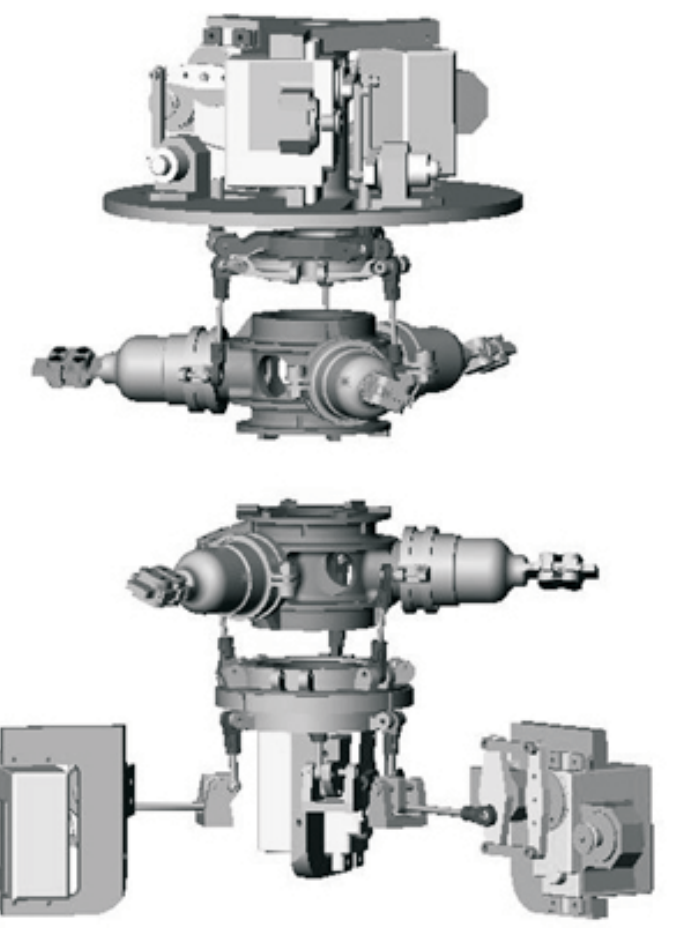

Fig. 5: Solid model of the mechanism for blade pitch control

with these tools. The field of mechanical design is probably the framework inside which the undergraduate students that took part to the research program obtained the greatest advantage in terms of new professional skills. On the other hand, it is only thanks to these tools, the use of which could be easily learned from self-contained manuals, that students with just a basic knowledge of mechanical design could successfully take part in a "real life" project, in spite of their lack of experience.

\section{Control system rapid prototyping}

After the realization of the rotor assembly and of the three-engine unit, which were separately ground tested, as reported in [4], ground testing of the whole engine/rotor system, coupled with the on-board computer for engine and rotor rpm control, will offer the first test benchmark for the control system software. As explained in greater detail in [7] and [13], the flight management system (FMS) is made of an inner robust control and stability augmentation system, coupled with basic autopilot functions, realized by external feedback loops. During normal operations, i. e., after the engine start-up procedure, the engine throttles are controlled by the so-called rpm governor, which is a simple SISO lead-lag compensator, with three poles and two zeros, with feedback on rotor speed, which must keep the rotor angular velocity at the nominal value of 3,000 rpm. Large gain (28.3 dB) and phase margins (103.4 deg) are required to avoid dynamic coupling between governor and UAV controller and to preserve the stability of the complete system (including the vehicle) due to the effect of several uncertainties and simplifications in the rotor dynamical model [14].
The FMS hardware architecture, described in [13], is based on a set of COTS PC/104 boards including

- No. 1 EuroTech 266 MHz CPU board with 64 Mb DRAM and a $32 \mathrm{Mb}$ disk-on-chip used to store user's programs and for data logging, 15 interrupt channels, 2 RS-232 serial ports: Executes flight control code, manages sensor data, organizes and commits telemetry to the ground station.

- No. 1 MESA Electronics 41234 channels RS-232 board: Is the interface with IMU/GPS main attitude/navigation sensor, digital compass and wireless modem.

- No. 2 Diamond System Corp. Quartz-MM 10 board with 8 digital input lines, 8 digital output lines and 10 counter/timers: Used to generate PWM signals for 12 coupled servos (6 output channels) controlling upper and lower swashplates, 3 servos for engine throttles, and 2 servos for roll/pitch video camera control, and to interface with 5 Hall-effect latches, RPM sensors for engines (3) and rotors (2).

- No. 1 Diamond System Corp. Diamond-MM 32 board with 32 single or 16 differential analog input channels (16 bit), 4 analog output channels (12 bit) and 24 digital I/O lines: Used for analog signal acquisition, that is, EGT and cylinder head temperatures, voltage and current in the power system.

- No. 1 EuroTech IDEA-NET2/ET 10 BaseT Ethernet board: Used to connect the FMS to a laptop computer or prior-to-flight system diagnosis and initialization.

- No. 1 Diamond Systems Corp. 50 Watt DC/DC power supply board.

Development of onboard SW is entirely carried out using the Mathworks Matlab/Simulink and Stateflow with Real-Time Workshop and Stateflow Coder as automatic code generation tools. The latter produce customizable C-code directly from Simulink and Stateflow diagrams for stand-alone embedded system applications. The xPC Target toolbox with xPC Target Embedded Option provide automatic implementation of executable code in the target computer, where an efficient real-time kernel is running. Supported hardware is connected to the Simulink models via the S-function input/output interface [15]. In this way, rapid prototyping of the control law synthesized in the Matlab/Simulink environment can be performed in the Hardware-In-The-Loop (HITL) simulation facility, the structure of which is depicted in Fig. 6.

Even if it is by far the simplest element of the control system, the reliability of the rpm governor is of paramount importance for the vehicle. Moreover, its demonstration will assess the viability of the approach and the HW configuration, based on the elements and the design tools listed above. For this reason, an experimental setup was designed in order to test the engine system under automatic control. In order to limit the risk of losing the prototype, or important parts of it because of a major, unpredictable failure, a safe approach was undertaken where the engine is coupled with an aerodynamic brake, made by a bar with adjustable rectangular panels, instead of the actual rotor system (Fig. 7). The brake was sized in order to create an aerodynamic torque $Q$ at the design speed $\Omega=3,000 \mathrm{rpm}$ reasonably close to that produced by the rotor blades. Using a simple strip theory for the 


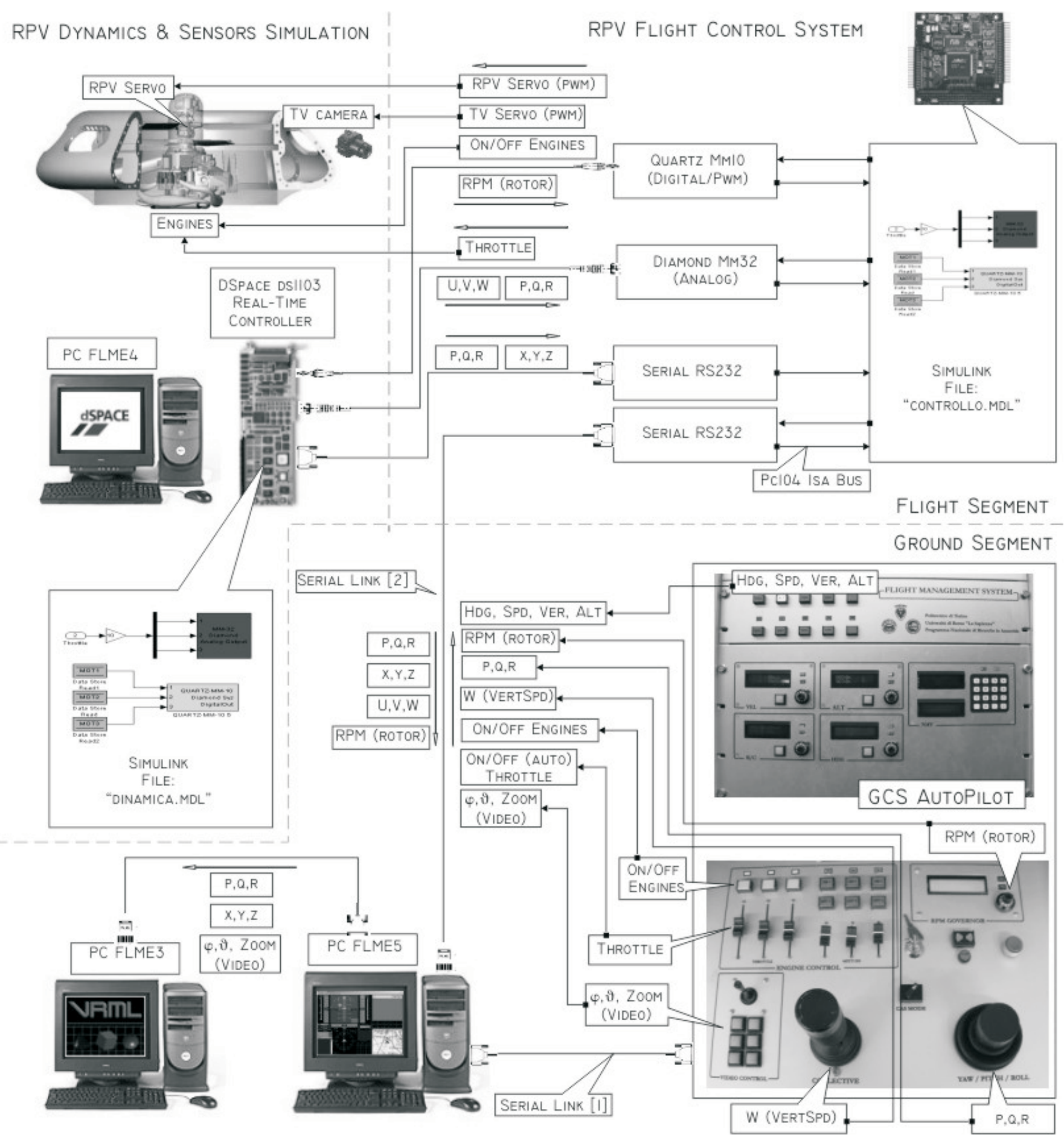

Fig. 6: Architecture of control system hardware in the HITL simulation facility

bar and assuming a drag coefficient $C_{\mathrm{DP}}$ for the two panels of surface $S_{\mathrm{P}}$, the torque estimate is given by

$$
Q=\rho \Omega^{2}\left(R_{\mathrm{P}}^{3} S_{\mathrm{P}} C_{\mathrm{DP}}+\int_{r_{\mathrm{h}}}^{R} r^{3} C_{\mathrm{D}} a \mathrm{~d} r\right)
$$

where $R_{\mathrm{P}}$ is the adjustable position of the panel, $2 R$ is the bar length, $a$ its thickness, and $2 r_{\mathrm{h}}$ is the diameter of the central hub. If the position of the panels is varied along the bar, the brake torque can be varied at will, in order to simulate different rotor loads.

At present, the on-board hardware has successfully demonstrated the possibility of managing manually all the main tasks related to engine operations [16]. The next step, i.e. to automatically manage throttle setting with the engine running, is much more ambitious, but it is also a crucial stage for the entire project, inasmuch as most of the hardware realized by the two research units will work assembled for the very first time in its definitive configuration. 


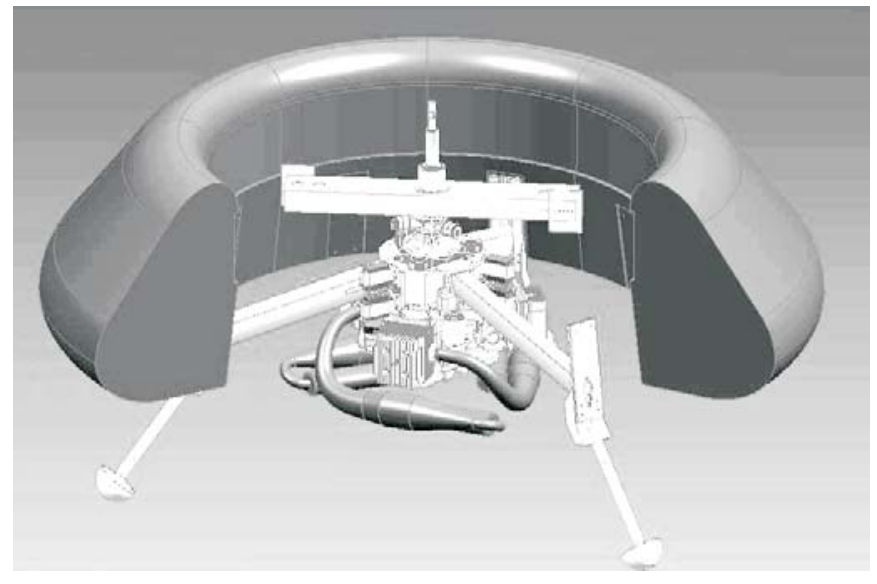

Fig. 7: Solid model of the prototype with the aerodynamic brake

\section{Conclusions and future work}

The key engineering technologies involved in the design and development of an uninhabited aerial vehicle in an academic environment were presented, with particular focus on the activities related to the development of the engine/rotor system. The paper describes in some detail how these advanced tools enabled academic staff, with contributions from undergraduate students, to complete the design of a wholly original machine, build the prototype and ground test its main components.

Work currently under way is aimed at demonstrating the overall viability of the approach undertaken for developing the control laws, starting from the simple SISO control system in charge of keeping a constant rpm level for the rotors. The success of this phase represents a key step towards the in-flight demonstration of the prototype, i.e., a demonstration of the whole UAV system.

\section{References}

[1] Koo, T. J. et al.: "Hybrid Control of an Autonomous Helicopter.” In: Proc. IFAC Workshop on Motion Control, Grenoble, France, September 1998.

[2] La Civita, M. et al.: "Design and Flight Testing of a High-Bandwidth H-infinity Loop Shaping Controller for a Robotic Helicopter.” AIAA Paper 2002-4836. In: Proc. AIAA Guidance, Navigation, and Control Conference and Exhibit, Monterey, CA, August 2002.

[3] Corban, J. E. et al.: "Flight Evalutation of Adaptive High-Bandwidth Control Methods for Unmanned Helicopters.” AIAA Paper 2002-4441. In: Proc. AIAA Guidance, Navigation, and Control Conference and Exhibit, Monterey, CA, August 2002.

[4] Avanzini, G., D’Angelo, S., de Matteis, G.: "Design and Development of a VTOL Uninhabited Aerial Vehicle." J. of Aerospace Eng., Vol. 217 (2003), No. 4, p. 169-178.

[5] Avanzini, G., D’Angelo, S., de Matteis, G.: "Modeling and Simulation of a Shrouded-Fan UAV for Environmental Monitoring." AIAA Paper 2002-3464, AIAA $1^{\text {st }}$ Tech. Conf. \& Workshop on UAV Systems, Technologies, and Operations, Portsmouth (VA), USA, May 2002.
[6] Avanzini, G., D’Angelo, S., de Matteis, G.: "Performance and Stability of a Ducted-Fan Uninhabited Aerial Vehicle.” J. of Aircraft, Vol. 40 (2003), No. 1, p. 86-93.

[7] Avanzini, G., Boserman, F., de Matteis, G.: "Design of a Rate Command m-Controller for a Shrouded-Fan Uninhabited Aerial Vehicle." AIAA Paper 2003-5521. In: Proc. of the AIAA Guidance, Navigation and Control Conference, Austin (TX), USA, August 2003.

[8] Lipera, L. et al.: "The Micro Craft iSTAR Micro Air Vehicle: Control System Design and Testing." Proc. of the Am. Helicopter Soc. $57^{\text {th }}$ Annual forum, Washington (DC), USA, May 2001.

[9] Thornberg, C. A., Cycon, J. P.: "Sikorsky Aircraft's Unmanned Aerial Vehicle, Cypher: System Description and Program Accomplishments." Proc. of the Annual Helicopter Society $51^{\text {st }}$ Annual Forum, Fort Worth (TX), USA, May 1995, p. 804-811.

[10] VSAERO User's Manual, Analytical Methods Inc., Rev. E5, Seattle (WA), USA, April 1994.

[11] de Divitiis, N.: "Aerodynamic Modeling and Performance Analysis of a Shrouded Fan Unmanned Aerial Vehicle." Proc. of the $23^{\text {rd }}$ Congress of the International Council of the Aeronautical Sciences, Paper 256, Toronto, Canada, September 2002.

[12] FINE/TURBO User Manual. Version 3.1, Numerical Mechanics Applications, Brussels, Belgium, March 1999.

[13] Avanzini, G., Boserman, F., de Matteis, G.: "Development of the Flight Management System for a Shrouded-Fan U. A. V.” Proc. of the XVII Congress of the Italian Society of Aeronautics and Astronautics, Rome, Italy, September 2003.

[14] Avanzini, G., de Matteis, G., Fresta, F.: "Robust Multivariable Control of a Shrouded-Fan Uninhabited Aerial Vehicle.” AIAA Paper 2002-4703. In: Proc. of the AIAA Atmospheric Flight Mechanics Conference, Monterey, CA, August 2002.

[15] Simulink: Dynamic System Simulation for Matlab. The Mathworks Inc., 1997.

[16] Avanzini, G., Badami, M., D’Angelo, S., de Matteis, G.: "Integration and Preliminary Testing of Avionic System and Engine Unit of a Twin Rotor U. A. V.” Proc. of the XVII Congress of the Italian Society of Aeronautics and Astronautics, Rome, Italy, September 2003.

Giulio Avanzini

e-mail: giulio.avanzini@polito.it

Salvatore D'Angelo

Department of Aerospace Engineering

Politecnico di Torino

C.so Duca degli Abruzzi, 24

Turin, 10129, Italy

Guido de Matteis

Department of Mechanics and Aeronautics

Universit di Roma "La Sapienza”

Via Eudossiana, 18

Rome, 00184, Italy 